\title{
Case - Management of bullet retained in penile shaft after drive-by shooting
}

\author{
Max Levitt, MD ${ }^{1}$; Thomas Ying, MD ${ }^{1}$; Ehab Abdalla Elzayat, $\mathrm{MD}^{1}$ \\ ${ }^{1}$ Division of Urology, Department of Surgery, The Ottawa Hospital, Ottawa, ON, Canada
}

Cite as: Levitt M, Ying T, Elzayat EA. Case - Management of bullet retained in penile shaft after driveby shooting. Can Urol Assoc J 2021 October 18; Epub ahead of print. http://dx.doi.org/10.5489/cuaj.7480

Published online October 18, 2021

Corresponding author: Dr. Ehab Elzayat, Division of Urology, Department of Surgery, University of Ottawa, Ottawa, ON, Canada; eelzayat@toh.ca

$$
* * *
$$

\section{Introduction}

Civilian trauma involving the penis is exceptionally rare, and even in high-volume trauma centers, only several cases involving penile gunshot are seen per year. ${ }^{1,2}$ Rarer still, are cases in which the projectile is retained in the penis. We present a case of penetrating penile trauma with a retained bullet in the base of the penis.

\section{Case report}

In May 2021, a 32-year-old male was involved in a drive-by shooting in which he was seated in the passenger seat of a vehicle struck by gunfire aimed the driver's side. The firearm used was presumed to be a handgun but this was unverified. He presented to our city's tertiary care trauma center in stable condition and a trauma code was initiated. During the trauma team's primary survey, six small superficial wounds were found in the suprapubic fat pad and left thigh. A Foley catheter was inserted by ER staff and entered the bladder without resistance, draining clear urine devoid of visible blood. A portable abdominal X-ray revealed a radioopaque foreign body in soft tissue of the pelvis and external genitalia (Fig. 1A). CT angiogram done as part of his trauma workup further characterized the foreign body as a $12 \mathrm{~mm}$ bullet fragment at the right aspect of the mid penile shaft (Fig. 1B). There was no visible vascular injury in the groin or left thigh. In addition, an $11 \mathrm{~mm}$ metallic bullet fragment was found adjacent to the left femur, without evidence of fracture. On physical exam, the circumcised penis appeared normal. A firm foreign body was palpable at the anterior base of the penile shaft. There was no cutaneous injury of the penis or penile hematoma. The scrotum appeared intact and uninjured with no edema or hematoma. The testes were not tender to palpation and were of normal consistency. No additional genitourinary or other organ injuries were noted. Based on imaging and physical exam findings, the patient was taken to the OR for urgent penile exploration. 


\section{Operative steps}

A subcoronal incision was made along the previous circumcision scar and the penis was degloved. Buck's fascia was incised over the palpable foreign body. The bullet fragment was located between the right and left corpus cavernosum, just inferior to the dorsal neurovascular bundle in the proximal third of the penile shaft (Fig. 2A). A tourniquet was applied to the base of the penis, and the somewhat deformed bullet fragment was removed intact (Fig. 2B). Further exploration revealed a 2 $\mathrm{cm}$ defect in the right medial tunica albuginea and a $1 \mathrm{~cm}$ defect in the left anteromedial tunica albuginea. These were repaired using 3/0 Maxon suture. Buck's fascia was re-approximated using $3 / 0$ Vicryl suture and the tourniquet was removed. No bleeding was observed from the corporeal bodies. Cystourethroscopy was performed, and the urethra appeared fully intact. No obvious injuries, blood clots, or foreign bodies were seen in the bladder. We then returned to closure where hemostasis was achieved, the penile skin replaced, and the circumcising incision re-approximated using $4 / 0$ Chromic suture. A Foley catheter was placed intraoperatively and removed prior to discharge on postoperative day 1 .

\section{Followup}

The patient was seen two weeks later in the ER due to complications from his thigh wound. At urologic assessment, the penile incision appeared well healed and there was no hematoma or palpable fibrosis. The patient reported good erectile function with minimal pain. The patient reported no penile paresthesia or loss of sensation.

\section{Discussion}

Only three other cases of retained bullets in the penis have been described in the literature. ${ }^{3-5}$ In one of these cases, the bullet was superficial and was removed at the bedside without formal penile exploration. ${ }^{3}$ The other two reports describe penile exploration with removal of the bullet and repair of the tunica albuginea. ${ }^{4,5}$

Due to the rarity of the described injury, specific guidelines pertaining to diagnosis and management are lacking. Generally accepted principles for managing penile penetrating trauma include: prompt penile exploration if corporeal injury is suspected, urethral evaluation with either cystoscopy or retrograde cystogram if there is blood at the urethral meatus, inability to void or gross hematuria, and repair of corporeal and/or urethral defects if encountered. ${ }^{6}$ The subcoronal degloving incision is the most commonly described surgical approach to penile exploration in the setting of penetrating trauma ${ }^{1}$. Penile degloving allowed adequate exposure in the described case, and prompted urethral evaluation once corporeal defects were encountered. Furthermore, several authors advocate for artificial erection testing to localize corporeal injury and assess for repair adequacy. ${ }^{4,7}$ This step was omitted in the described case as the brisk bleeding that was encountered prior to the application of a penile tourniquet stopped entirely after repair.

A retained bullet fragment in the penis may be missed if comprehensive imaging is not performed at presentation. In a similar case, diagnosis was delayed for several days as an upfront CT 
scan was not performed. ${ }^{5}$ Furthermore, early surgical exploration and reconstruction of the corpora cavernosa may prevent the development of undesired late complications, such as urethral stricture, penile deformity/curvature, and erectile dysfunction. ${ }^{6,89}$ In our case, initial management by the trauma team included pelvic X-ray which revealed the presence of a retained bullet in the suprapubic region. A CT scan further localized it to the base of the penis, prompting surgical exploration, and revealed an additional bullet in the left thigh. Indeed, up to $90 \%$ of civilian gunshot injuries to the genitals have concomitant organ injuries, most commonly in the lower limb and scrotum. ${ }^{1}$ Taken together, the importance of cross-sectional imaging in genital gunshot injury is paramount.

The American Association for the Surgery of Trauma (AAST) injury severity score is useful for facilitating uniform treatment of traumatic injury to the genitalia. ${ }^{10}$ Contusions and superficial lesions are classified as grade I and can be conservatively treated. Higher grades of injury include: grade II (Buck fascia lacerations without tissue loss), grade III (cutaneous avulsion or laceration through glands and meatus, or urethral or cavernosum lesions less than $2 \mathrm{~cm}$ in area), grade IV (partial penectomy or urethral or cavernosum lesions more than $2 \mathrm{~cm}$ in area), and grade $\mathrm{V}$ (total penectomy). The AAST states that injury grades II-V require surgical exploration, ${ }^{10,11}$ however, some authors advocate for conservative management for both grade I and II injuries. ${ }^{12}$ In our case, due to the suspicion of corporeal injury on imaging, we estimated the injury as an AAST grade III or IV. This injury severity was confirmed upon surgical exploration.

\section{Conclusions}

Penile gunshot wounds are rare traumatic injuries. Rarer still are cases in which the bullet is retained in the penis. While specific guidelines for this scenario do not exist, we advocate for evaluation with detailed physical exam, cross-sectional imaging, and retrograde urethrogram or cystogram. Urgent penile exploration for removal of foreign body, debridement of devitalized tissue, and primary repair of corporeal defects is the preferred management strategy. The AAST injury severity score is another useful tool for guiding management. 


\section{References}

1. Cerwinka WH, Block NL. Civilian gunshot injuries of the penis: the Miami experience. Urology 2009;73:877-80.

2. Kunkle DA, Lebed BD, Mydlo JH, et al. Evaluation and management of gunshot wounds of the penis: 20-year experience at an urban trauma center. J Trauma 2008;64:1038-42.

3. Kumar M, Parmar KM, Attri V, et al. Bullet in penis: uncommon site, unusual track and unexpected result. Urology \& Nephrology Open Access Journal 2020;8.

4. Raheem AA, Alowidah I, Almousa M, et al. Management of a Retained Bullet in the Corpora Cavernosa after a Civilian Gunshot Injury: A Rare Scenario. J Trauma Inj 2020;33:275-78.

5. Shirol S, Nerli R, Prabha V, Nimbaragi G. Bullet injury to the penis with retained bullet in the penis. International Journal of Pharma Medicine and Biological Sciences 2012;1:33-6.

6. Morey AF, Brandes S, Dugi DD, et al. Urotrauma: AUA guideline. J Urol. 2014;192:327-35.

7. Gopal N, Schwalb D. Isolated injury to the left distal corpus cavernosum from a through-andthrough penile gunshot wound. Urology Case Reports 2019;27:100918.

8. Cavalcanti AG, Krambeck R, Araujo A, et al. Penile lesion from gunshot wound: a 43-case experience. Int Braz J Urol 2006;32:56-60

9. Bozzini G, Albersen M, Otero JR, et al. Delaying Surgical Treatment of Penile Fracture Results in Poor Functional Outcomes: Results from a Large Retrospective Multicenter European Study. European Urology Focus 2018;4:106-10.

10. Moore EE, Malangoni MA, Cogbill TH, et al. Organ Injury Scaling VII: Cervical Vascular, Peripheral Vascular, Adrenal, Penis, Testis, and Scrotum. Journal of Trauma and Acute Care Surgery 1996;41:523-24.

11. Morey AF, Metro MJ, Carney KJ, et al. Consensus on genitourinary trauma: external genitalia. BJU Int 2004;94:507-15.

12. Mohr AM, Pham AM, Lavery RF, et al. Management of trauma to the male external genitalia: the usefulness of American Association for the Surgery of Trauma organ injury scales. J Urol 2003;170:2311-15. 


\section{Figures and Tables}

Fig. 1. (A) Pelvic X-ray showing radio-opaque foreign body in the suprapubic region. (B) Computed tomography scan showing foreign body located at the base of the penile shaft. Arrows point to the foreigh body.

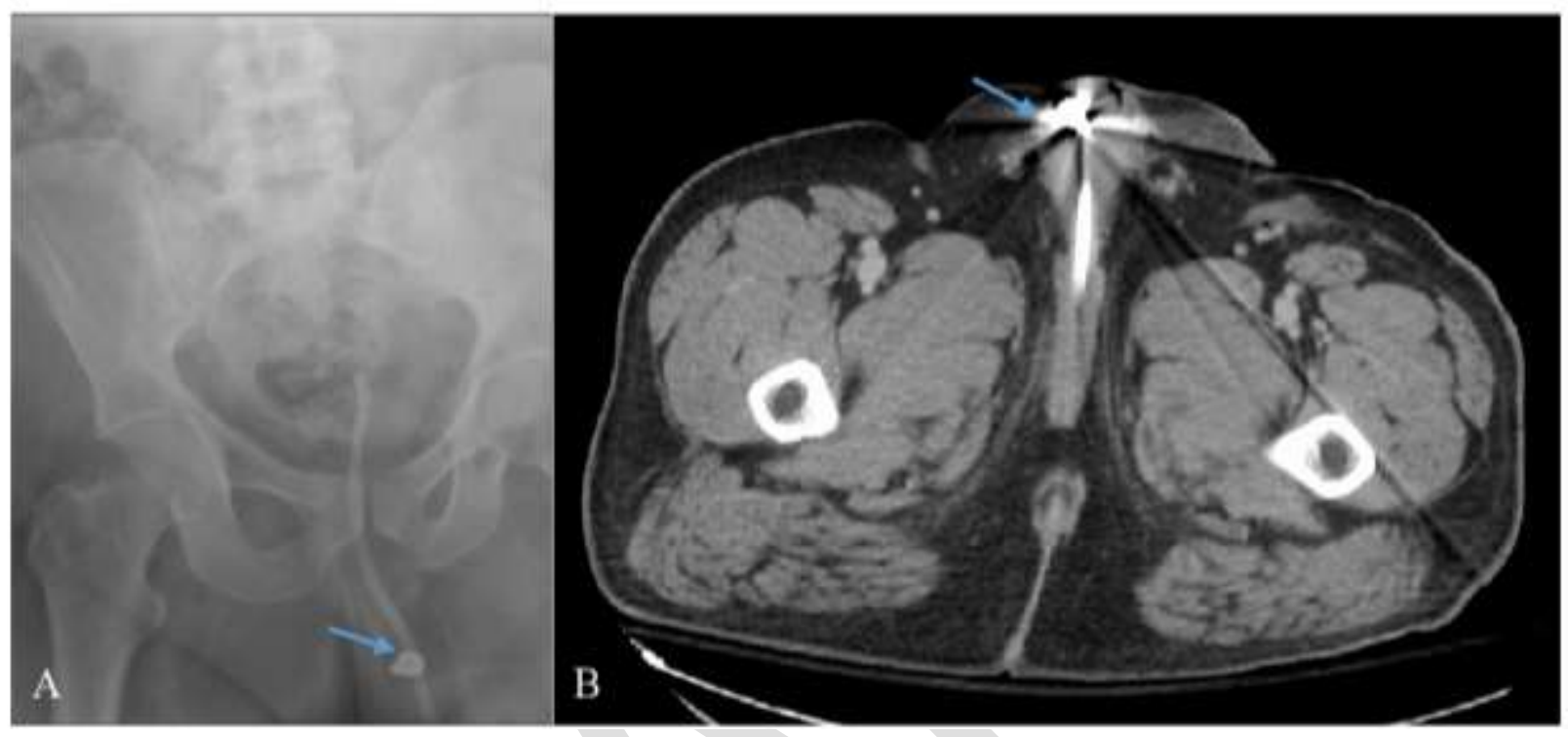

Fig. 2. (A) Intraoperative image showing bullet retained within corpora cavernosa (arrow), deep to and not involving the neurovascular bundle (surrounded by vessel loop). (B) Intact and slightly deformed bullet.

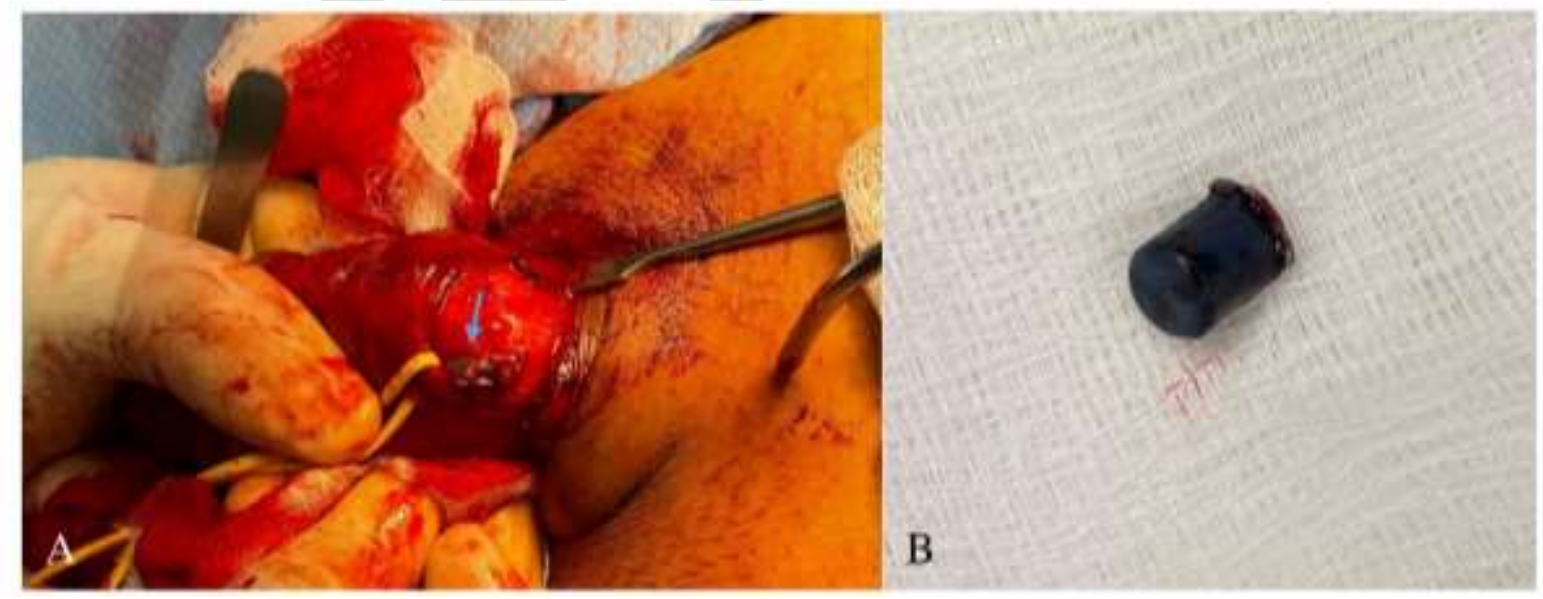

